\title{
Capacitive immunosensor for C-reactive protein quantification
}

\author{
Christos Sapsanis, Shilpa Sivashankar, Hesham Omran, Ulrich Buttner, Khaled Nabil Salama \\ Sensors Lab, Electrical Engineering Program \\ King Abdullah University of Science and Technology (KAUST), Thuwal 23955-6900, Saudi Arabia. \\ khaled.salama@kaust.edu.sa
}

\begin{abstract}
We report an agglutination-based immunosensor for the quantification of C-reactive protein (CRP). The developed immunoassay sensor requires approximately 15 minutes of assay time per sample and provides a sensitivity of $0.5 \mathrm{mg} / \mathrm{L}$. We have measured the capacitance of interdigitated electrodes (IDEs) and quantified the concentration of added analyte. The proposed method is a label free detection method and hence provides rapid measurement preferable in diagnostics. We have so far been able to quantify the concentration to as low as $0.5 \mathrm{mg} / \mathrm{L}$ and as high as $10 \mathrm{mg} / \mathrm{L}$. By quantifying CRP in serum, we can assess whether patients are prone to cardiac diseases and monitor the risk associated with such diseases. The sensor is a simple low cost structure and it can be a promising device for rapid and sensitive detection of disease markers at the point-of-care stage.
\end{abstract}

Keywords-interdigitated electrodes, immunoassay sensor, CRP quantification, agglutination, biosensor, capacitive sensor, cardiac marker.

\section{INTRODUCTION}

Immunoassays are crucial and versatile analytical tools with a wide range of application in the field of clinical diagnostics, forensics and biomolecular research. Researchers make concerted efforts to detect ultrasensitive [1], label-free [2] simple and high throughput [3] assays. Small amounts of biomarkers are detected via quantitative immunoassays in physiological fluids [4]. These assays are used in detecting environmental contaminants [5] and infectious or toxic substances [6] and monitoring therapeutic drugs [7]. Traditional assays like cards and slides or conventional assays that are usually performed in micro well plates have several shortfalls that include complexities in fluids handling, requirement for large sample volumes and increased assay time. These demerits have limited the use of traditional and conventional systems in diagnostics [8]-[10]. Another disadvantage of these systems is the need for fluorescent labeling, which is tedious and time consuming practice. Therefore developing a device that can save time and simplify the process of sample preparation could be useful at the point-of-care.

The biosensors area is considered an emerging field. Although, a multitude of instruments is used as biosensors, they can be found mainly in labs. There is only one wellknown example of a low-cost portable point of care device; the glucose sensor [11]. Thus, this research area is fuelled by the need for portability, miniaturization and low-cost biosensors in order to avoid the usage of expensive equipment, which also requires a special user or doctor increasing the cost even further. Electrochemical biosensors are capable of directly transforming the biological information into an electrical signal. This fact leads to a less complex setup in the information extraction. Moreover, this biosensor type is robust and compatible with microelectronic circuits leading to cost and size decrease.

Quantitative analysis of certain proteins is crucial in clinical applications as it aids in diagnosing specific diseases. One such diagnostic protein is CRP, which was discovered in 1930 by Tillett and Francis [12]. These investigators identified a substance in the blood of patients with acute pneumococcal pneumonia that reacted with the cell wall C-polysaccharide of the bacteria. It is an acutephase serum protein and a member of the pentraxin protein family. It has a mass of $120 \mathrm{kDa}$ and it is composed of five identical polypeptide subunits held together by noncovalent interactions. Its host defense functions predate the adaptive immune system by millions of years. The structure of CRP has been determined by X-ray crystallography at $3 \AA$ resolution [13][14]. The overall dimensions of the CRP pentamer are about $102 \AA$ outside diameter with a central pore diameter of $30 \AA$ and a protomer diameter of $36 \AA$. CRP is an inflammation and cardiac disease marker. Thus, detecting it is crucial because various concentrations present in human serum indicate an increased risk or even the presence of cardiac disease [15].

Capacitive immunoassays are promising alternatives to immunochemical tests for the development of point-of-care devices. The analytes are bound to the antibody latex reagent that had been immobilized on the electrode surface. The change in capacitance, when the analytes bind to the antibody complex, is measured and this entire set up is typically termed as capacitive biosensors. By measuring the ratio of the capacitance in the final measurement to the first one before the process starts, we obtain an indication of the analyte. This ratio can be simplified into the relative permittivity of the anti-CRP antibody and CRP antigen interaction film since this is the primary reason for this capacitance difference. We have developed a capacitive immunosensor for accurate, rapid and cost-effective diagnosis of diseases at the point-of-care.

This work cannot be directly compared to the cases of [16]-[18] from the results perspective since a Network analyzer (NA) has been used. The main goal in our measurements is to achieve reliable capacitance readout. The LCR meter usage in the current work is used as a proof of concept for CRP quantification, which can pave the way for a fully integrated microsystem by using a simple IC circuitry for capacitance readout [19]-[21]. This cannot occur in the case of the NA, which is a complicated and expensive instrumentation typically present in a laboratory setting. Moreover, in our case, we took advantage of agglutination phenomena for forming the complex, which has significantly simplified the experimental process. Thus, simple instrumentation and fast procedure can have potentials for a portable application. 


\section{MATERIALS AND METHODS}

\section{A. Interdigitated electrodes (IDEs) Fabrication}

The IDEs were fabricated in the KAUST Nanofab using a 4-inch $<100>$ p-type silicon wafer of $2.5-4.0 \Omega \cdot \mathrm{cm}$ electrical resistivity from MEMC. A 2 um oxide layer was thermally grown for electrical isolation. This oxidized silicon wafer was selected to imitate IDEs grown in a standard CMOS process. The IDEs were patterned using a subtractive process, which gives better results compared to lift-off, given the thin and long IDEs fingers.

First, $10 \mathrm{~nm} \mathrm{Ti}$ and $300 \mathrm{~nm}$ Au metal layers were deposited via physical vapor deposition (PVD) for $60 \mathrm{~s}$ and 360 s respectively in an ESC reactive and metal sputter system. Ti was used as an adhesion layer rather than $\mathrm{Cr}$ because it is more easily dry etched. The selection of $\mathrm{Au}$ is based on its favorable chemical and electrical characteristics. For both metals, the rest recipe is the same: $400 \mathrm{~W}$ of DC power, $25 \mathrm{sccm}$ of Ar flow, pressure of 5 mTorr and pre-sputtering time of $30 \mathrm{~s}$.

In the next step, optical lithography was used to pattern the electrodes. An image reversal photoresist (AZ5124E) was spun at $3000 \mathrm{rpm}$ for $30 \mathrm{~s}$ yielding a $1.6 \mathrm{um}$ photoresist layer, followed by soft baking at $105^{\circ} \mathrm{C}$ for $120 \mathrm{~s}$. A 5 -inch dark-field optical mask was created using a Heidelberg uPG101 Laser Mask Writer. The photoresist was patterned using contact lithography and a $90 \mathrm{~mJ} / \mathrm{cm}^{2}$ broadband exposure dose. For image reversal, the wafer was then heated again at $120{ }^{\circ} \mathrm{C}$ for $120 \mathrm{~s}$ followed by flood exposure. Puddle developing for $60 \mathrm{~s}$ using AZ726 was performed in SUSS MicroTec Delta 12AQ spray develop system.

The metal layer was patterned by dry etching using the Oxford Instruments PlasmaLab System. A $30 \mathrm{sccm}$ flow of Ar was used for 4 min with $150 \mathrm{~W}$ RF power and $1000 \mathrm{~W}$ ICP power. The exposed oxide thickness was further verified using the Nanospec 6100 Reflectometer to ensure that the metal layer was properly etched. Dry etching prevented the acetone from completely stripping the photoresist layer and thus $\mathrm{O}_{2}$ plasma had to be used. The $\mathrm{O}_{2}$ plasma step also partially etches the oxide between the IDEs fingers. This enhances the sensitivity because the agglutination complex fills completely the region between the fingers, which increases the maximum electric field. The IDEs were designed with 4 um finger width and 3 um spacing. For our cleanroom photolithography tools, this was the minimum reliable feature that could provide high capacitance.

Finally, the capacitive structure was coated with a $350 \mathrm{~nm}$ thin film of Parylene $\mathrm{C}$ by chemical vapor deposition (CVD). The coating was conformal with the thickness to be controllable by the recipe and the inserted Parylene $\mathrm{C}$ powder. In our case, 0.6 gram of Parylene $\mathrm{C}$ powder was used in order to achieve the thickness of $350 \mathrm{~nm}$. This step enhanced the capacitors isolation and kept its dissipation factor to acceptable levels after depositing the analyte. Schematic representation of the fabricated IDEs cell in 2D and 3D using an optical microscope and the Nanosurf Easyscan 2 AFM are illustrated in Fig. 1 and Fig. 2 respectively. The whole cleanroom fabrication process is depicted in Fig. 3.

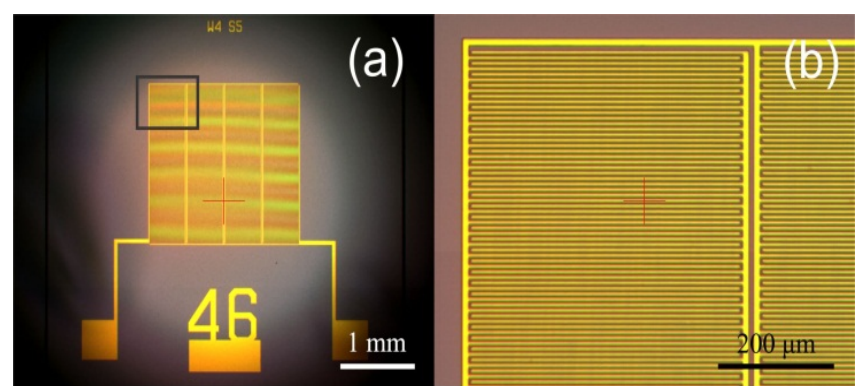

Fig. 1. (a) Optical microscope image of the fabricated IDE device; (b) enlarged image of enclosed area in (a).

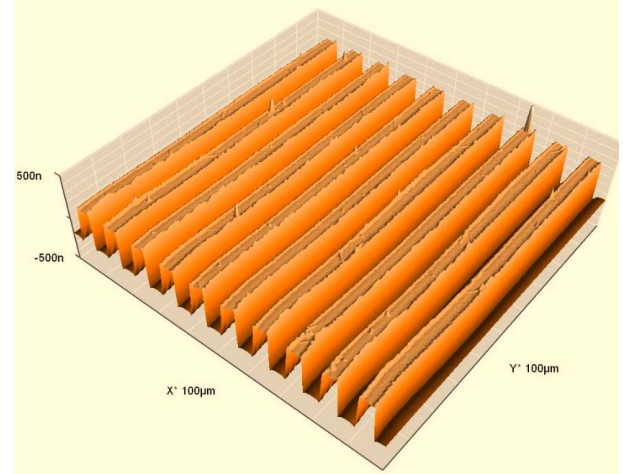

Fig. 2. Fabricated IDEs in 3D by Nanosurf Easyscan 2 AFM

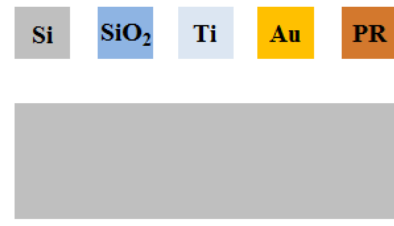

(a) Bare Si wafer

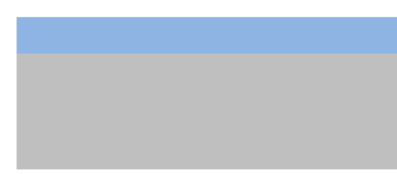

(b) Thermal Growth of $2 \mathrm{um} \mathrm{SiO}_{2}$

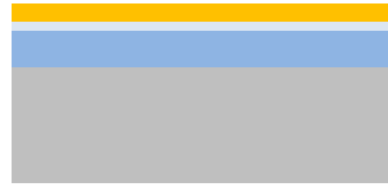

(c) Sputtering of $10 \mathrm{~nm}$ Ti and $300 \mathrm{~nm} \mathrm{Au}$

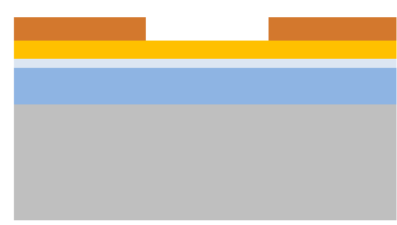

(d) Pattering (Lithography) of PR

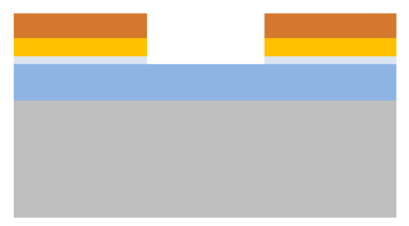

(e) Dry etch

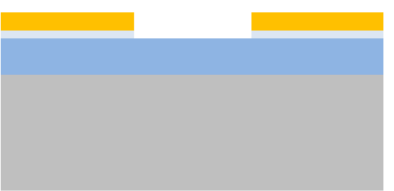

(f) $\mathrm{O}_{2}$ Descum (Ashing) of PR

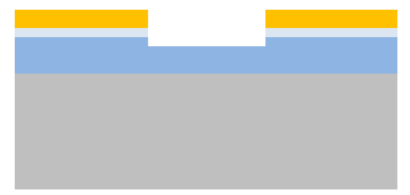

(g) RIE etch of oxide

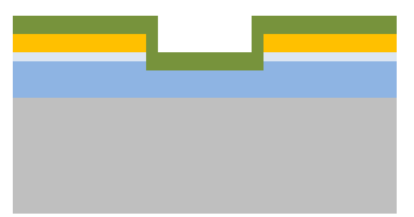

(h) CVD of $350 \mathrm{~nm}$ Parylene C
Fig. 3. Fabrication process of interdigitated electrodes.

\section{B. Experimental Procedure}

From the biological sensor definition, it is an analytical device that converts a biological response into an electrical signal by combining a biological component with a physicochemical detector. In our case, the transducer was a gold IDEs array coated with $350 \mathrm{~nm}$ of Parylene C. Efforts have been made to improve upon agglutination assays for their potential in detecting biomarkers. Biomarkers provide 
insight into normal biological processes, pathogenic processes and pharmacological therapeutic interventions. The agglutination process occurs when anti-CRP antibodies interact with CRP protein (antigen). In general, agglutination takes place when an antigen is in a mixture with its corresponding antibody called isoagglutinin. The degree of agglutination depends on the concentration of antigen added to a specific concentration of antibody and vice versa. The whole process is illustrated in Fig. 4.

Anti-CRP antibodies coated on the latex beads were employed throughout the experiments. Commercially available CRP (Applied Chem) of human serum was diluted to obtain various concentration of CRP. At first the antibodies were immobilized and then the antigens were introduced to form an agglutination complex. A precision pipette was used for introducing the complex and evenly distributing it across the IDEs. The capacitance was measured throughout the experiment duration. The main focus was on the values before the antibody immobilization and following the point where the complex was almost agglutinated, when the capacitance was in its highest value. An LCR meter (Keysight E4980a) was utilized at a frequency of $1 \mathrm{MHz}$ at $1 \mathrm{~V}$.
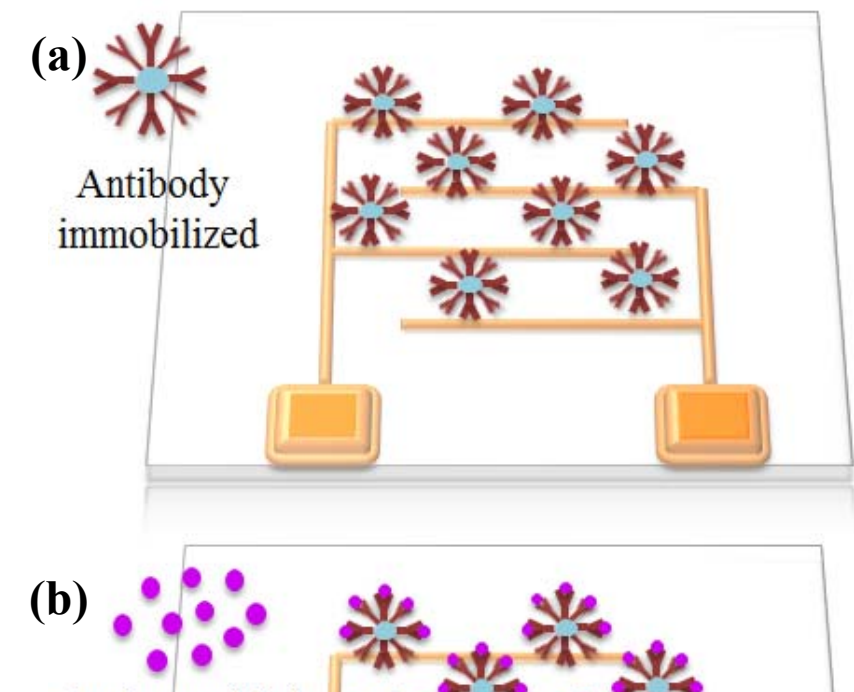

Antigen added

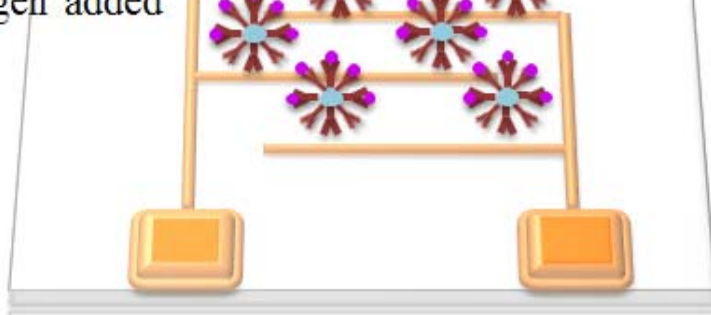

Fig. 4. Agglutination Process (a) antibody being immobilized (b) antigens added and captured by antibodies

The overall capacitance in the readout of the sensor can be modeled, as also depicted in Fig. 5, by a fringing and a sidewall capacitance between the electrodes through the agglutination complex. There is also a background offset fringing capacitance through $\mathrm{SiO}_{2}$ and $\mathrm{Si}$, which can be considered equal for all the test samples since the same fabrication process was followed.

Cleaning has been conducted to the samples using DI $\mathrm{H}_{2} \mathrm{O}$ to investigate any possibility for reusability. The main obstacle for reusing was the fact that the Parylene $\mathrm{C}$ layer has been affected leading the isolation to degrade. Thus, new samples were used for each trial in order to obtain reliable and comparable results throughout the experiments with the different CRP concentrations.

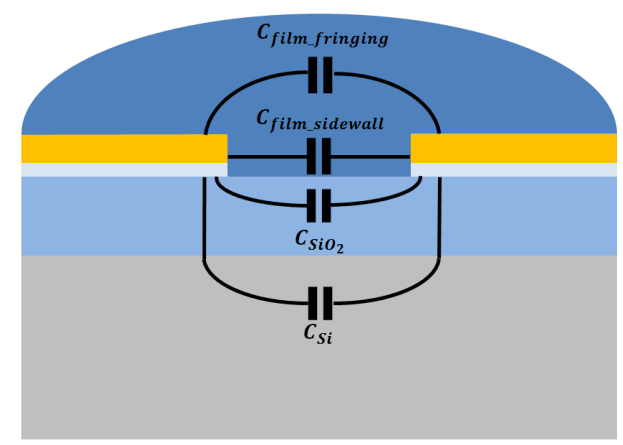

Fig. 5. Types of capacitance constituting the overall capacitor

\section{RESUlTS AND DISCUSSION}

After the immobilization of the anti-CRP antibodies, the IDEs capacitance increased as expected due to the dielectric constant of the antibody. The same fact occurred after the antigen injection, constituting the agglutination complex. This enhancement is based on the fringing and sidewall field that passes through the complex.

The results indicated the process of agglutination started immediately after analyte introduction and agglutination was progressing with time. For instance, agglutination with change over time for the concentration of $2 \mathrm{mg} / \mathrm{L}$ is depicted using film images in Fig. 6a. The changes of agglutination complex with time are represented by measuring the capacitance in Fig. 6b. During this process up until it is fully agglutinated, the capacitance value showed a slow increasing trend in the graph. This behavior was predictable since the antibodies are clumping antigens and the material properties vary. After reaching the highest value, the complex was completely formed. The capacitance value is decreased since evaporation occurred and caused changes in the properties of the mixture.

The concentrations were chosen based on the risk at which a patient can be prone to cardiac disease. According to the American Heart Association (AHA) and the United States Center for Disease Control (CDC), a CRP concentration below $1.0 \mathrm{mg} / \mathrm{L}$ represents low risk; a range from 1.0 to $3.0 \mathrm{mg} / \mathrm{L}$ represents medium (average) risk; a measurement over $3.0 \mathrm{mg} / \mathrm{L}$ represents high risk. Thus, the concentrations of $0.5 \mathrm{mg} / \mathrm{L}, 2 \mathrm{mg} / \mathrm{L}, 4 \mathrm{mg} / \mathrm{L}$ and $10 \mathrm{mg} / \mathrm{L}$ were selected as these assess the risk according to the aforementioned limits.

Analytes were added and the measured capacitance gave an indication of the analyte concentration, with an average of three trials per sample. The relative permittivity versus the antigen concentration is plotted in Fig. 7. The detection limit for CRP detection is found to be $0.5 \mathrm{mg} / \mathrm{L}$, which applies to the low risk limits. We see for the $0.5 \mathrm{mg} / \mathrm{L}, 2$ $\mathrm{mg} / \mathrm{L}$ and $4 \mathrm{mg} / \mathrm{L}$ of analyte concentration that the relative permittivity $\left(\mathrm{e}_{\mathrm{r}}\right)$ increases. On the other hand, there is a slight decrease in the $\mathrm{e}_{\mathrm{r}}$ from $4 \mathrm{mg} / \mathrm{L}$ to $10 \mathrm{mg} / \mathrm{L}$. This fact is based on the Hook effect. Hook effect is a common phenomenon in agglutination assays wherein it shows false negative results even in the presence of excess analyte, which has also occurred in [22] for CRP quantification. More specifically, the excess amount of antigen in the sample cannot be bound with the coated antibodies and this leads to no agglutination. Therefore, it behaves as if a lower 
concentration were added, which explains the decrease in capacitance value corresponding to $10 \mathrm{mg} / \mathrm{L}$ of sample concentration compared to $4 \mathrm{mg} / \mathrm{L}$.

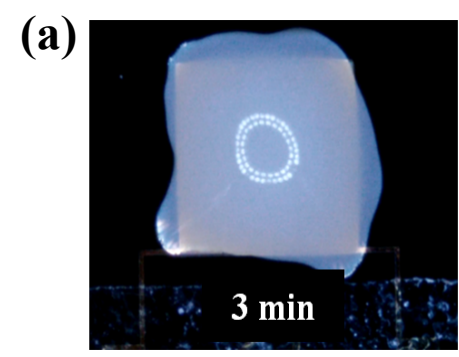

(i) Initial Stage

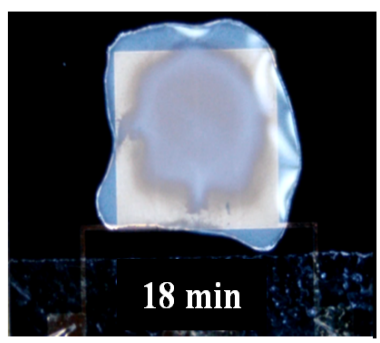

(iii) Almost agglutinated and evaporation initiates

(b)

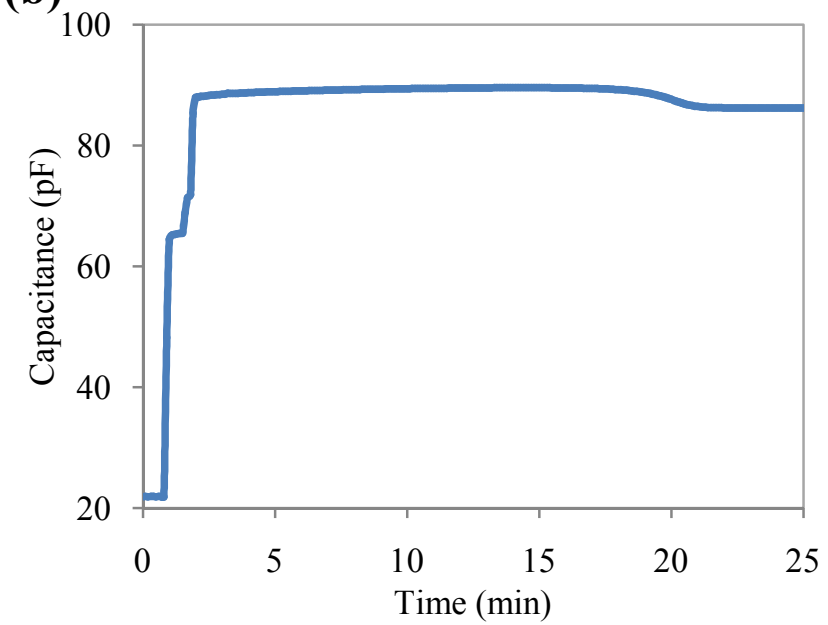

Fig. 6. Agglutination progressing with time in (a) film images and (b) capacitance versus time diagram.

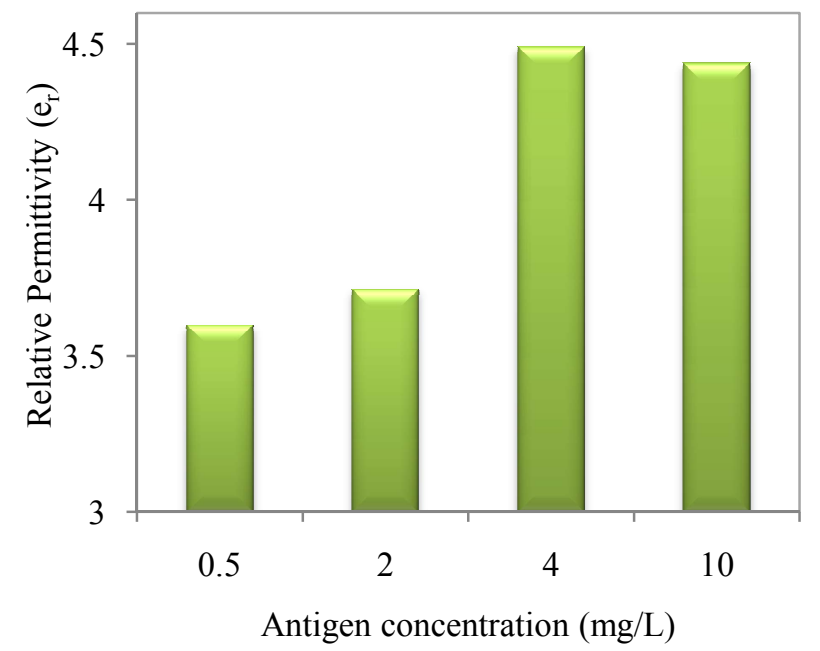

Fig. 7. Relative Permittivity versus Antigen concentration.

\section{CONCLUSION}

In conclusion, we have implemented a simple and low cost capacitive structure for the quantification of C-reactive protein. The proposed immunoassay sensor requires a simple sample preparation process, is fast and not tedious, which is favorable for the early detection of a given sample in diagnostics. It has been proven that the concentration of CRP can be distinctly differentiated in the limit of $3 \mathrm{mg} / \mathrm{L}$, which acts as a biomarker of cardiac disease risk. These sensors also show hook-effect that is in harmonious agreement with the normal biological process proving that these sensors can be used in point-of-care devices.

\section{REFERENCES}

[1] Yang, Yi-Ning, et al. "An integrated microfluidic system for Creactive protein measurement." Biosensors and Bioelectronics 24.10 (2009): 3091-3096.

[2] Okuno, Jun, et al. "Label-free immunosensor for prostate-specific antigen based on single-walled carbon nanotube array-modified micro electrodes." Biosensors and Bioelectronics 22.9 (2007): 2377-2381.

[3] Wang, Jing, et al. "Colorimetric multiplexed immunoassay for sequential detection of tumor markers." Biosensors and Bioelectronics 25.2 (2009): 532-536.

[4] Ekins, Roger. "Immunoassay: recent developments and future directions." Nuclear medicine and biology 21.3 (1994): 495-521.

[5] Sia, Samuel K., et al. "An Integrated Approach to a Portable and Low-Cost Immunoassay for Resource-Poor Settings." Angewandte Chemie International Edition 43.4 (2004): 498-502.

[6] Hatch, Anson, et al. "A rapid diffusion immunoassay in a T-sensor." Nature biotechnology 19.5 (2001): 461-465.

[7] Cho, Joung-Hwan, et al. "Plastic ELISA-on-a-chip based on sequential cross-flow chromatography." Analytical chemistry 78.3 (2006): 793-800

[8] Dodge, Arash, et al. "Electrokinetically driven microfluidic chips with surface-modified chambers for heterogeneous immunoassays." Analytical chemistry 73.14 (2001): 3400-3409.

[9] Sato, Kiichi, et al. "Determination of carcinoembryonic antigen in human sera by integrated bead-bed immunoasay in a microchip for cancer diagnosis." Analytical Chemistry 73.6 (2001): 1213-1218.

[10] Gao, Yali, et al. "Development of a novel electrokinetically driven microfluidic immunoassay for the detection of Helicobacter pylori." Analytica chimica acta 543.1 (2005): 109-116.

[11] Grieshaber, Dorothee, et al. "Electrochemical biosensors-sensor principles and architectures." Sensors 8.3 (2008): 1400-1458.

[12] Tillett, William S., and Thomas Francis. "Serological reactions in pneumonia with a non-protein somatic fraction of pneumococcus." The Journal of experimental medicine 52.4 (1930): 561-571.

[13] Shrive, Annette K., et al. "Three dimensional structure of human Creactive protein." Nature Structural \& Molecular Biology 3.4 (1996): 346-354.

[14] Thompson, Darren, Mark B. Pepys, and Steve P. Wood. "The physiological structure of human C-reactive protein and its complex with phosphocholine." Structure 7.2 (1999): 169-177.

[15] Lee, Wen-Bin, et al. "An integrated microfluidic system for fast, automatic detection of C-reactive protein." Sensors and Actuators B: Chemical 157.2 (2011): 710-721.

[16] Qureshi, Anjum, et al. "Label-free capacitive biosensor for sensitive detection of multiple biomarkers using gold interdigitated capacitor arrays." Biosensors and Bioelectronics 25.10 (2010): 2318-2323.

[17] S.S. Kallempudi, et al. "Label-free biosensors for the detection and quantification of cardiovascular risk markers", Sens. Lett. 6 (2008)

[18] Quershi, Anjum, et al. "A novel interdigitated capacitor based biosensor for detection of cardiovascular risk marker." Biosensors and Bioelectronics 25.4 (2009): 877-882.

[19] Omran, Hesham, Muhammad Arsalan, and Khaled N. Salama. "An integrated energy-efficient capacitive sensor digital interface circuit." Sensors and Actuators A: Physical 216 (2014): 43-51.

[20] Omran, Hesham, Muhammad Arsalan, and Khaled N. Salama. "A 7.9 $\mathrm{pJ} /$ Step Energy-Efficient Multi-Slope 13-bit Capacitance-to-Digital Converter." (2014): 1-1.

[21] Omran, Hesham, Muhammad Arsalan, and Khaled N. Salama. "A robust parasitic-insensitive successive approximation capacitance-todigital converter." Custom Integrated Circuits Conference (CICC), 2014 IEEE Proceedings of the. IEEE, 2014.

[22] Byun, Ju-Young, et al. "A colorimetric homogeneous immunoassay system for the C-reactive protein." Analyst 138.5 (2013): 1538-1543. 